\title{
COVID-19 and Myocarditis: Findings from Cardiac Magnetic Resonance Imaging and Endomyocardial Biopsies
}

\author{
Simon Greulich ${ }^{1} \quad$ Karin Klingel ${ }^{2}$ \\ ${ }^{1}$ Department of Cardiology and Angiology, University of Tübingen, \\ Tübingen, Germany \\ ${ }^{2}$ Cardiopathology, Institute for Pathology and Neuropathology, \\ University of Tübingen, Tübingen, Germany \\ Address for correspondence Simon Greulich, MD, Department of \\ Cardiology and Angiology, University of Tübingen, Otfried Müller \\ Strasse 10, Tübingen 72074, Germany \\ (e-mail: Simon.greulich@med.uni-tuebingen.de).
}

Hamostaseologie 2021;41:366-370.

\begin{abstract}
Keywords

- COVID-19

- myocarditis

- CMR

- histology/ immunohistology

- virus infection

Diagnosing myocarditis is still challenging due to its varying presentation ranging from none or mild symptoms to sudden cardiac death. Clinical presentation, electrocardiography, and cardiac biomarkers seem not to be sufficient for a reliable diagnosis. In fact, an unequivocal myocardial characterization is needed, applying endomyocardial biopsy (EMB) and cardiac magnetic resonance (CMR), a technique which demonstrates high accuracy to histology. Besides the assessment of functional parameters (volumes, ejection fraction), established late gadolinium enhancement and recent T1 and T2 mapping techniques including the calculation of extracellular volume fraction allow distinct myocardial tissue analysis by a noninvasive approach without the need of radiation. However, EMB is the only method which allows the identification of the underlying etiology of cardiac inflammation. Since myocardial damage and inflammation seem to be prevalent in a considerable number of patients even in the mid-term range after COVID-19, CMR and EMB seem to be adequate tools to further investigate these findings. In this article, we will (1) review current knowledge about the role of CMR in the COVID-19 pandemic and (2) report about our own EMB findings in COVID-19 patients in the Cardiopathology Center of our University Hospital.
\end{abstract}

\section{Introduction}

Recent publications suggest an association of severe acute respiratory syndrome coronavirus 2 (SARS-CoV-2) infection with increased mortality in patients with and without preexisting cardiovascular diseases. Older age ( $>60$ years), male sex, and the presence of comorbidities, including hypertension and obesity, have been identified as risk factors for death in patients with coronavirus disease (COVID)-19. ${ }^{1,2}$ Despite a strong association of the severity of COVID-19 and myocardial injury, the exact mechanisms of myocardial injury in these patients are not well understood. The suggested mechanisms of myocardial damage are most likely

received

May 11, 2021

accepted after revision

June 27, 2021 due to nonischemic myocardial processes, including severe respiratory infection with hypoxia, sepsis, pulmonary thrombosis and embolism, activation of the immune system as a consequence of the release of multiple inflammatory mediators (cytokines), and possibly myocarditis. ${ }^{3}$ However, the question how many patients with COVID-19 really suffer from histologically proven acute and chronic myocarditis is not solved.

Due to its heterogeneous presentation, diagnosis, monitoring, and prediction of events in patients with myocarditis are still challenging. ${ }^{4,5}$ Although endomyocardial biopsy (EMB) is still the gold standard, multi-parametric cardiac magnetic resonance (CMR) has gained increasing attention $\begin{array}{ll}\text { (c) 2021. Thieme. All rights reserved. } & \text { DOI https://doi.org/ } \\ \text { Georg Thieme Verlag KG, } & \text { 10.1055/a-1539-8664. } \\ \text { Rüdigerstraße 14, } & \text { ISSN 0720-9355. } \\ \text { 70469 Stuttgart, Germany } & \end{array}$ 
as a noninvasive tissue characterization approach in the diagnosis (and prediction of adverse events) in patients with suspected myocarditis. Specifically, subtle myocardial changes which might be detected by recent mapping techniques in early, potential reversible stages of the disease, would be of high clinical interest, suggesting a closer monitoring of these patients. Therefore, findings of CMR might also be of clinical value for COVID-19 in which patients seem to show a tendency for ongoing myocardial injury and inflammation during the further course of the disease.

Here, we present current findings of (1) CMR as a noninvasive imaging tool for myocarditis in COVID-19 and (2) EMB results of patients with COVID-19.

\section{Role of Cardiac Magnetic Resonance in COVID-19 Myocarditis}

Recently, CMR criteria for the diagnosis of acute myocarditis were updated, acknowledging the emerging role of newer techniques such as quantitative T1 and T2 mapping and extracellular volume fraction (ECV). These latter techniques seem to refine the diagnosis of myocarditis in the detection of even subtle myocardial alterations besides the established late gadolinium enhancement (LGE) technique, with increased T1 values indicating myocardial fibrosis or inflammation, and increased $\mathrm{T} 2$ values indicative of myocardial edema occurring in inflammatory heart disease. ECV indicates expanded extracellular space by fibrotic or inflammatory processes. 5,6

A systematic review including 199 patients with COVID19 in whom CMR was performed suggests that myocarditis is the most prevalent diagnosis (in $40.2 \%$ of patients) detected by a combination of $\mathrm{T} 1$ and $\mathrm{T} 2$ mapping values (with the highest percentage of abnormalities as 73 and 63\%, respectively), and LGE (43\%). ${ }^{7}$ Interestingly, ventricular function was preserved in most patients, underlining the unmet need of further myocardial tissue characterization by CMR. Here, recent mapping techniques seem adequate in the detection of diffuse myocardial changes, as irreversible fibrotic (or necrotic) changes indicated by extensive areas of LGE are not so common in the present literature (also see - Fig. 1).

The largest cohort so far investigated 100 patients recently recovered from COVID-19 illness by a comprehensive CMR approach. Here, cardiac involvement as demonstrated by abnormal CMR parameters was demonstrated in 78 patients (78\%), and ongoing inflammation in 60 patients $(60 \%){ }^{8}$ Findings were reported without considering preexisting conditions, severity and overall course of the acute illness, and time from original diagnosis. Of note, native T1 mapping and T2 mapping demonstrated the best discriminatory value for the detection of COVID-19-related cardiac abnormalities compared with healthy controls. ${ }^{8}$ Unfortunately, only three of these patients underwent EMB due to the presence of severe myocardial abnormalities (increased troponin, native $\mathrm{T} 1+\mathrm{T} 2$, presence of LGE, and impaired left ventricular ejection fraction), revealing active lymphocytic inflammation without evidence of viral genome.

Another study confirmed the high percentage of cardiac abnormalities detected by CMR parameters in COVID-19

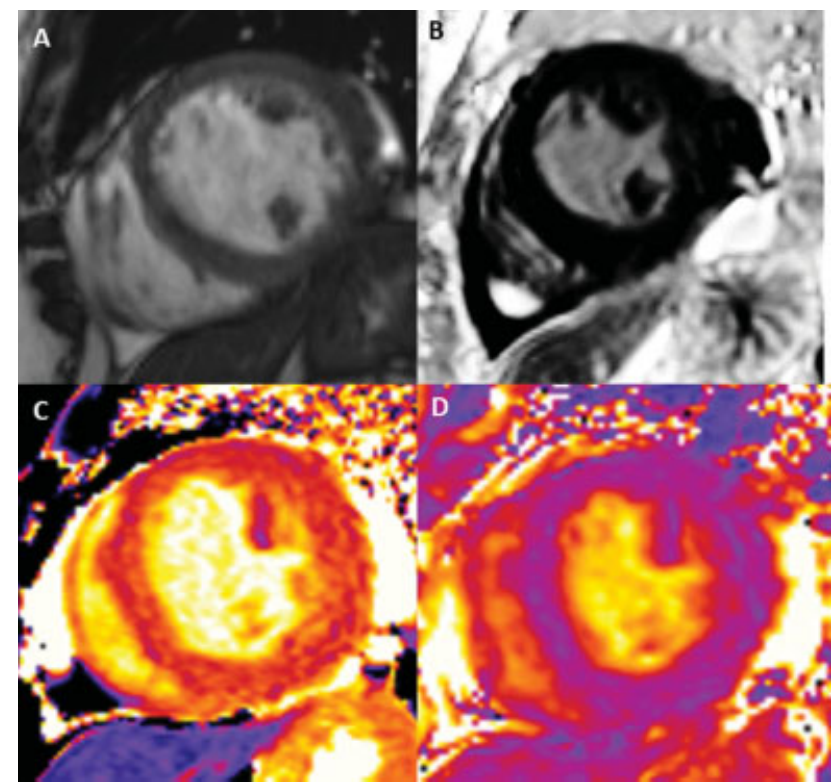

Fig. 1 A 46-year old COVID-19-positive female presenting with recurrent chest pain and dyspnea under exertion. Echocardiography revealed severely impaired left ventricular ejection fraction (30\%), pericardial effusion. Coronary artery disease was ruled out by X-ray coronary angiography. CMR images demonstrate an enlarged left ventricle (A), absence of LGE (B), and (C) increased native T1 values (1,300 ms) suggesting diffuse myocardial fibrosis/edema, and (D) increased T2 value (52 ms), suggesting diffuse myocardial inflammation.

patients: Huang et al analyzed retrospectively CMR data from 26 patients who recovered from COVID-19 including LGE, T2-weighted imaging, and recent mapping techniques (T1, T2, ECV). 58\% showed abnormal conventional CMR findings (mirrored by increased T2 signal and/or positive subepicardial or midwall LGE). In these patients, mapping values (native T1, T2, and ECV) were significantly increased compared with patients without positive "conventional" CMR findings and healthy controls, ${ }^{9}$ suggesting a high prevalence of diffuse myocardial involvement as a consequence of COVID-19.

\section{Conclusions of Cardiac Magnetic Resonance in COVID-19}

The reported CMR studies underline a high percentage of cardiac involvement, suggesting myocarditis-like processes in COVID-19 patients during the acute phase of SARS-CoV-2 infection which seem to persist in a considerable number of patients during the further course of the disease. Therefore, COVID-19 patients should be closely monitored by the treating physician regarding symptoms and signs of heart failure. CMR with its unique strength of detailed noninvasive tissue characterization can facilitate the detection of cardiac involvement especially in subtle cases who may present with unspecific symptoms as well as inconspicuous echocardiography and electrocardiography. Further larger studies should investigate the value of CMR parameters not only for diagnosis but also for the prediction of adverse events in COVID19 and post-COVID-19 patients. 


\section{Endomyocardial Biopsy Results in COVID-19}

Primarily on the basis of laboratory (elevation of troponin levels) and imaging data (CMR), ${ }^{9,10}$ myocarditis has been assumed to play a role in the pathogenesis of COVID-19; however, it is unclear how many patients with SARS-CoV-2 infection have histologically/immunohistologically proven acute and chronic myocarditis. To evaluate whether cardiac inflammation (i.e., myocarditis) plays a role in COVID-19, we performed the workup of EMBs from 88 patients (15-91 years) having a history of proven SARS-CoV-2 infection as diagnosed by positive polymerase chain reaction in the throat, swab, sputum, or serum of patients. EMBs were obtained between 4 days and 3 months after the diagnosis of SARS-CoV-2 infection. The majority of patients were male ( $n=66$, median age: 51.35 years), and 22 patients were female (median age: 59.11 years). All patients had the clinical diagnosis of COVID-19 and suspicion of myocarditis by echocardiography and CMR with elevated blood biomarkers such as troponin $\mathrm{T}$ and $\mathrm{I}$ and $\mathrm{N}$-terminal pro-B-type natriuretic peptide.

EMBs were investigated by histology using hematoxylin and eosin (H\&E) and Giemsa stains to identify cardiac injury and the patterns of inflammation according to the criteria presented by Caforio et al. ${ }^{11}$ Immunohistochemical staining was performed to detect CD3 $+\mathrm{T}$ cells and CD68+ macrophages by using automated diagnostic staining devices. Fibrosis was assessed in Masson Trichrome-stained tissue sections (-Fig. 2). Virus infections including SARS-CoV-2 were evaluated by (qRT-)PCR in the myocardium.

In histological stainings, the majority of patients did not reveal acute myocardial damage (i.e. myocyte necrosis). Only 8 of 66 male and 2 of 22 female patients revealed the criteria of acute lymphocytic myocarditis with myocyte necrosis and 30 to $150 \mathrm{CD} 3+\mathrm{T}$ cells $/ \mathrm{mm}^{2}$ and significantly increased amounts of $\mathrm{CD} 68+$ macrophages. One patient had acute
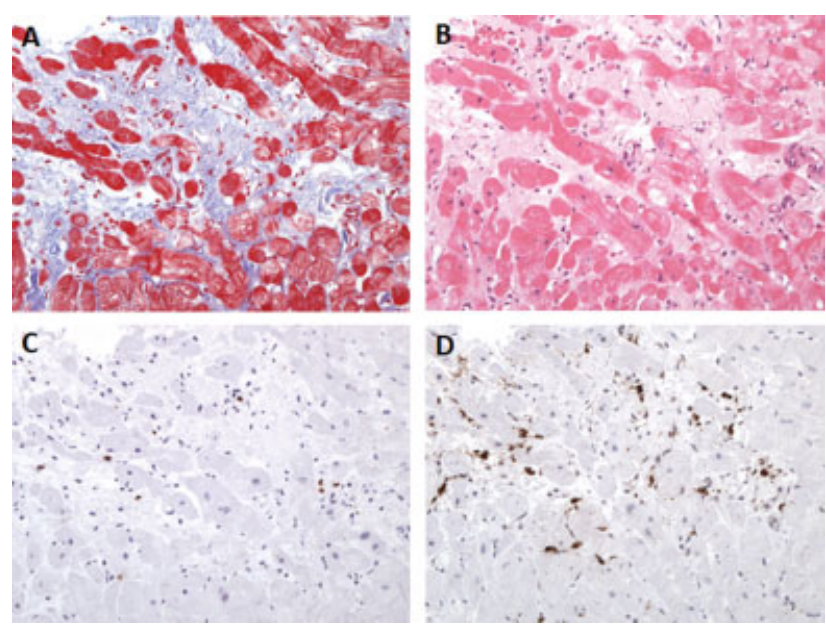

Fig. 2 EMB images of a 46-year old female patient (see - Fig. 1) with acute SARS-CoV-2 infection and healing chronic lymphocytic

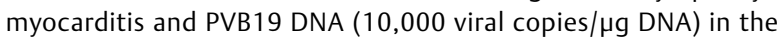
myocardium. (A) Masson Trichrome and (B) HE staining reveals fibrosis, (C) infiltration of CD3 $+\mathrm{T}$ cells, and (D) CD68+ macrophages (x200). eosinophilic myocarditis. In none of the patients with acute myocarditis we found other cardiotropic viruses (e.g., enteroviruses, adenoviruses, herpesviruses (Epstein-Barr virus [EBV], human herpesvirus 6 [HHV6], cytomegalovirus). No relevant interstitial fibrosis was observed. In 15 male and 10 female patients, we found chronic/healing myocarditis with 7 to $30 \mathrm{CD} 3+\mathrm{T}$ cells $/ \mathrm{mm}^{2}$ and increased amounts of macrophages $\left(>20 / \mathrm{mm}^{2}\right)$ in EMB. In 4 of 15 male patients with this diagnosis we found in the myocardium nucleic acids of other cardiotropic infections (HHV6, EBV, and PVB19 (parvovirus B19)). In 1 of 10 female patients PVB19 DNA (10,000 viral copies/ug cardiac DNA) was detected (see - Fig. 2), and in 1 of 10 female patients we found cardiac EBV DNA. All 25 patients demonstrated different degrees of interstitial fibrosis. In 70 of 88 COVID-19 patients, we observed increased amounts of interstitial macrophages. Only 16 male and 2 female patients with past SARS-CoV-2 infection and previously known heart diseases including dilated cardiomyopathy, toxic cardiomyopathy, hypertensive heart disease, noncompaction cardiomyopathy had no increased numbers of macrophages or $\mathrm{T}$ cells and thus, myocarditis was not prevalent in these patients. In the majority of patients, an interstitial fibrosis was noted.

In SARS-CoV-2-positive patients with suspected myocarditis and increased amounts of interstitial CD68+ macrophages, we confirmed the diagnosis of other cardiovascular diseases by evaluation of EMB in 27 male patients: dilated cardiomyopathy $(n=13)$, ischemic heart disease $(n=3)$, cardiac amyloidosis $(n=4)$, hypertensive heart disease $(n=1)$, hypertrophic cardiomyopathy $(n=1)$, toxic cardiomyopathy $(n=1)$, and other cardiac diseases including tachymyopathy $(n=4)$. Eight female patients revealed comorbidities including dilated cardiomyopathy $(n=3)$, amyloidosis $(n=1)$, other heart diseases including noncompaction cardiomyopathy ( $n=4$; - Fig. 3 ).

Interestingly, only three patients revealed SARS-CoV-2 RNA in the myocardium including a male patient with the histological diagnosis of acute eosinophilic myocarditis and another male patient with healed myocarditis without inflammation. The SARS-CoV-2 RNA-positive female revealed dilated cardiomyopathy and increased amounts of macrophages. We did not observe endothelial cell death in association with inflammation, indicating endotheliitis as previously published in autopsy cases ${ }^{12}$ or microthrombosis in EMBs of our patients.

\section{Conclusions of Endomyocardial Biopsy in COVID-19}

Our EMB results do not allow the conclusion that patients suffer more frequently from myocarditis in the course of SARS-CoV-2 infection than patients without this infection. In the same time frame, we had 77 cases with acute myocarditis and 1,960 cases with healing/chronic myocarditis (mostly lymphocytic myocarditis) which were not COVID-19 associated. It has to be stressed that in the majority of publications of COVID-19 patients with suspected myocarditis, no histology/immunohistology and molecular analyses, revealing the presence of other infections with cardiotropic viruses, were performed. Thus, the published findings of a high 


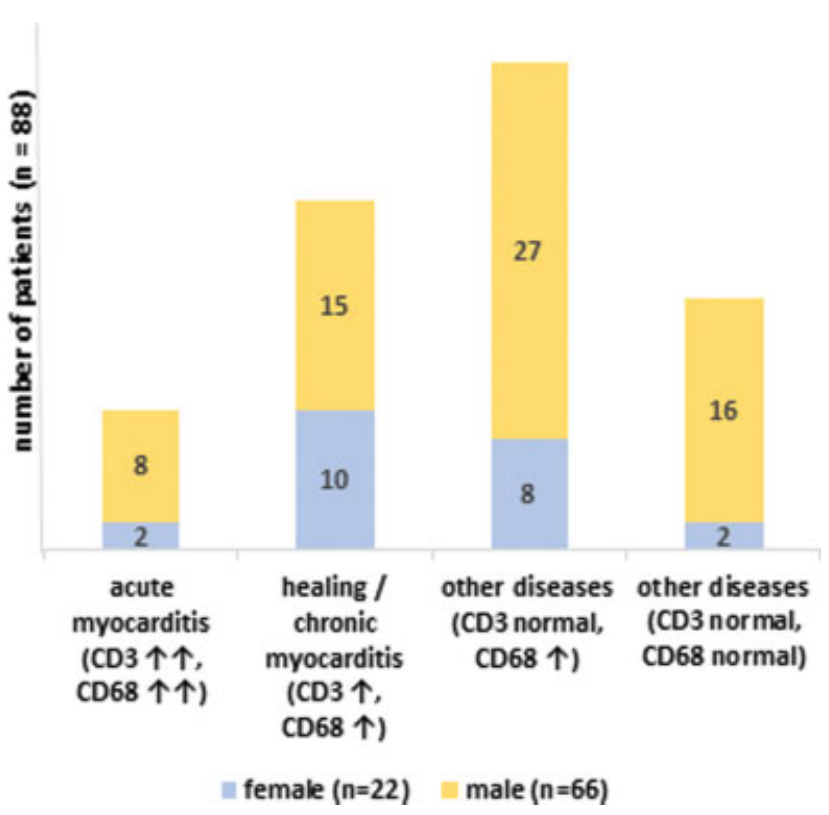

Fig. 3 EMB results of patients with COVID-19 history $(n=66$ males, $n=22$ females). EMB revealed acute myocarditis $(n=10)$, healing/chronic myocarditis $(n=25)$, increased amounts of CD68+ macrophages in patients with other cardiovascular diseases $(n=35)$, no infiltration of $\mathrm{CD} 3+\mathrm{T}$ cells or $\mathrm{CD} 68+$ macrophages in patients with other cardiovascular diseases $(n=18)$.

prevalence of acute cardiac damage by noninvasive methods such as CMR do not prove that SARS-CoV-2 is really inducing myocarditis, a disease which is defined by the presence of interstitial inflammatory cells as detected by immunohistochemistry in the myocardium. ${ }^{11}$ The rather low prevalence of histologically proven acute and chronic myocarditis in our cohort suggests that myocarditis does not play a major role among the suggested causes of myocardial injury in the course of COVID-19 disease. In none of the patients with acute lymphocytic myocarditis, we found SARS-CoV-2 RNA in the EMB. Also, considering the different time points of EMB (up to 3 months) after the diagnosis of SARS-CoV-2 infection in our patients, the absence of myocarditis in the majority of our patients does not support the idea of a persistent cardiac virus infection mediating chronic inflammation at later stages of infection. On the other hand, direct cytotoxic effects by the virus itself have been discussed. Despite the fact that we observed some virions in cardiomyocytes, ${ }^{13}$ endothelial cells or macrophages by electron microscopy (data not shown), a typical virus-induced cell lysis in association with lymphocytic infiltrates was never observed in EMBs in comparison to other RNA virus infections including coxsackieviruses. ${ }^{14}$ Importantly, it has to be considered that CMR results as well as the histological/immunohistological findings have to be interpreted on the background of the comorbidities of the patients which might reveal diseases such as ischemic cardiomyopathy, tachymyopathy, or toxic cardiomyopathy, which may also be associated with increased amounts of macrophages and increased expression of cytokines. ${ }^{15,16}$ Thus, further investigations are required to evaluate the contribution of SARS-CoV-2 in cardiac damage, especially with regard to the systemic cytokine generation and the specific role of macrophages which are elevated in the EMB of the majority of our COVID19 patients.

\section{Clinical Implications}

Recent CMR data suggest a high percentage of cardiac abnormalities in patients with (post) COVID-19, possibly induced by nonischemic alterations including myocarditis. However, the exact role of SARS-CoV-2 in the pathogenesis of cardiac inflammatory injury is not yet proven, as in our evaluation the majority of COVID-19 patients (53/88) did not reveal acute or chronic myocarditis in EMB. Thus, so far unknown molecular mechanisms seem to play a role in the COVID-19-associated heart disease, which should be elucidated by further studies combining the strengths of EMB and CMR, carefully considering the individual clinical information and comorbidities of the patients.

\section{Funding}

K.K. received basic funding from the Ministry of Science, Research and Arts of the State of Baden-Württemberg (COVID-19 Funding) and from Deutsche Herzstiftung.

\section{Conflict of Interest}

The authors declare that they have no conflict of interest.

\section{References}

1 Wang D, Hu B, Hu C, et al. Clinical characteristics of 138 hospitalized patients with 2019 novel coronavirus-infected pneumonia in Wuhan, China. JAMA 2020;323(11):1061-1069

2 Zheng YY, Ma YT, Zhang JY, Xie X. COVID-19 and the cardiovascular system. Nat Rev Cardiol 2020;17(05):259-260

3 Imazio M, Klingel K, Kindermann I, et al. COVID-19 pandemic and troponin: indirect myocardial injury, myocardial inflammation or myocarditis? Heart 2020;106(15):1127-1131

4 Greulich S, Seitz A, Müller KAL, et al. Predictors of mortality in patients with biopsy-proven viral myocarditis: 10-year outcome data. J Am Heart Assoc 2020;9(16):e015351

5 Greulich S, Ferreira VM, Dall'Armellina E, Mahrholdt H. Myocardial inflammation - Are we there yet? Curr Cardiovasc Imaging Rep 2015;8(03):6

6 Ferreira VM, Schulz-Menger J, Holmvang G, et al. Cardiovascular magnetic resonance in nonischemic myocardial inflammation: expert recommendations. J Am Coll Cardiol 2018;72(24): 3158-3176

7 Ojha V, Verma M, Pandey NN, et al. Cardiac magnetic resonance imaging in coronavirus disease 2019 (COVID-19): a systematic review of cardiac magnetic resonance imaging findings in 199 patients. J Thorac Imaging 2021;36(02):73-83

8 Puntmann VO, Carerj ML, Wieters I, et al. Outcomes of cardiovascular magnetic resonance imaging in patients recently recovered from coronavirus disease 2019 (COVID-19). JAMA Cardiol 2020;5 (11):1265-1273

9 Huang L, Zhao P, Tang D, et al. Cardiac involvement in patients recovered from COVID-2019 identified using magnetic resonance imaging. JACC Cardiovasc Imaging 2020;13(11):2330-2339

10 Zaccone G, Tomasoni D, Italia L, Lombardi CM, Metra M. Myocardial involvement in COVID-19: an interaction between comorbidities and heart failure with preserved ejection fraction. a further indication of the role of inflammation. Curr Heart Fail Rep 2021;18(03):99-106 
11 Caforio AL, Pankuweit S, Arbustini E, et al; European Society of Cardiology Working Group on Myocardial and Pericardial Diseases. Current state of knowledge on aetiology, diagnosis, management, and therapy of myocarditis: a position statement of the European Society of Cardiology Working Group on Myocardial and Pericardial Diseases. Eur Heart J 2013;34(33):2636-2648, 2648a-2648d

12 Varga Z, Flammer AJ, Steiger P, et al. Endothelial cell infection and endotheliitis in COVID-19. Lancet 2020;395(10234):1417-1418

13 Bojkova D, Wagner JUG, Shumliakivska M, et al. SARS-CoV-2 infects and induces cytotoxic effects in human cardiomyocytes. Cardiovasc Res 2020;116(14):2207-2215
14 Klingel K, Pöml P, Strunk J, Werthmann ML. Lethal enterovirus myocarditis in a patient with granulomatosis with polyangiitis following rituximab and high-dose steroid therapy. Eur Heart J 2021;42(24):2401

15 Mueller KAL, Heinzmann D, Klingel K, et al. Histopathological and immunological characteristics of tachycardia-induced cardiomyopathy. J Am Coll Cardiol 2017;69(17):2160-2172

16 Lutgens E, Atzler D, Döring Y, Duchene J, Steffens S, Weber C. Immunotherapy for cardiovascular disease. Eur Heart J 2019;40 (48):3937-3946 